\title{
Life on the Road: Exposing Drivers' Tendency to Anthropomorphise In-Vehicle Technology
}

\author{
David R. Large ${ }^{[0000-0003-3046-4984]}$ and Gary Burnett ${ }^{[0000-0001-9945-9396]}$ \\ Human Factors Research Group, University of Nottingham, Nottingham, UK \\ david.r.large@nottingham.ac.uk
}

\begin{abstract}
Anthropomorphism is often used in the design of products and technology, with the aim of enhancing the user experience. However, 'human' elements may also be employed for practical reasons, e.g. using speech as an interaction mechanism to minimise visual/manual distraction while driving. A selfreport questionnaire survey (attracting 285 respondents from the UK), enriched by over thirteen hours of ethnographic-style observations involving 14 participants, explored drivers' tendency to anthropomorphise a routine in-vehicle navigation device (employing speech to deliver instructions). While the self-reported behaviour of drivers revealed only limited evidence of anthropomorphism, the observations clearly demonstrated that such behaviour was abundant during everyday use, with plentiful examples of drivers and passengers assigning gender, names and personality to the device. Drivers also attempted to engage the device in conversation, apparently endowing it with independent thought, and blamed it for mistakes. The results raise important considerations for the design and development of future in-vehicle technology (where speech is employed as an interaction mechanism), and speech-based systems more widely.
\end{abstract}

Keywords: Anthropomorphism, Navigation Device, Survey, Ethnography.

\section{$1 \quad$ Introduction}

Drivers are increasingly presented with novel technological solutions to augment or replace manual aspects of the driving task. The willingness to engage with such technology is not only contingent on objective factors, such as the reliability and performance of such systems, but is also shaped by subjective perceptions [1]. This means that a driver's preferences and usage patterns are likely to be based on individual attitudes, expectations and experience [2], although these factors need not be directly related to functionality. Given recent trends towards the 'humanising' of interactions with technology (with exemplars such as Siri, Alexa and Cortana), an area that is increasingly of interest is the impact of anthropomorphism on users' attitudes and behaviour.

Anthropomorphism is defined as the "attribution of human motivation, characteristics, or behaviour to inanimate objects, animals, or natural phenomena" [3]. According to social and psychological theory, humans engage in anthropomorphism for various reasons, including familiarity, comfort, as a 'best-bet' solution, or to make sense of, or exert authority over an object [4]. The term is often used pejoratively in science, when 
behaviours considered exclusive to humans are attributed to non-human entities. Nevertheless, people's desire to anthropomorphise appears to be highly compelling, and there is an abundance of rich evidence across cultures and time, with contemporary examples including: interpreting the behaviour of pets sympathetically using human emotions, declaring the personality of a car based on its physical appearance, and coaxing or threatening a recalcitrant computer $[5,6]$. In these examples, exponents apparently use their experiences of 'being human' to help explain and guide their interactions with the nonhuman entity. Moreover, this occurs in spite of objective knowledge to the contrary [7]. Nevertheless, directing anthropomorphic attention towards certain subjects (e.g. pet animals, cartoon characters etc.) is generally considered to be more acceptable than attention towards other entities, such as cars and technology [8].

Anthropomorphism has been used extensively in design, with examples ranging from physical adornments to expressions of behavioural qualities [9]. Anthropomorphic designs are said to comfort users by providing clues about the product's function and mode of use, avoiding uncertainty and ambiguity, and facilitating certain social modes of interaction [10], but can also invite people to attach different personality traits to the host, or associate personal and social significance with it [4]. Anthropomorphism can therefore play a significant role in shaping user preferences [11]. However, using anthropomorphism in design can be problematic: it not only emphasizes the similarities but also hides the differences, and this can confuse or mislead users and create unrealistic expectations [12].

\section{$1.1 \quad$ Speech}

The inclusion of 'human' elements (e.g. speech) for pragmatic reasons may also result in anthropometric interpretations. One of the quintessential markers of humanness, speech is the primary means of social identification amongst humans [13]. From early infancy, humans are able to differentiate speech-like sounds from other sounds in their environment, and use this to maintain a sense of presence, even when their visual field is absent or obscured [14]. As humans develop and grow, they rapidly acquire the ability to extract salient, socially-relevant, paralinguistic cues from speech [13] based on vocal characteristics such as pitch, cadence, speech rate and volume, and use these to provide systematic and highly compelling guidance for determining gender, personality and emotion-specific actions [15]. Extensive research has demonstrated that humans appear to lack the wherewithal to overcome these evolutionary instincts, and behave towards vocal utterances from a computer - under experimental conditions at least - in a similar manner, ascribing humanlike characteristics and attending to talking machines as if they were interacting with another human $[15,16]$. Manipulating speech interfaces has therefore enabled researchers to exploit these automatic responses. For example, different digital 'personalities', created by varying the vocal characteristics and language content of spoken language interfaces, have been shown to influence trust, performance, learning and even consumers' buying habits during research studies [15].

From a pragmatic point of view, however, speech-based interfaces also afford 'handfree' and 'eyes-free' interaction, and are therefore often favoured by designers of computers or interfaces intended for deployment in situations already posing high levels of 
visual and/or manual demand, such as driving [17]. There is a substantial body of evidence which shows that using speech-based interfaces in vehicles (rather than traditional visual/manual methods of interaction) improves driving performance, reduces workload and passive task-related fatigue, and keeps drivers' eyes on the road [17,18]. However, as speech-based interfaces become more 'natural' (due to steady, sustained improvements in speech synthesis and natural language delivery), drivers' attitudes towards the technology (and its impact on their behaviour) may be influenced by subtle paralinguistic cues that define factors such as gender and personality, and create the illusion of human presence.

\subsection{Overview of Study}

To explore driver's tendency to attribute human motivation, characteristics, or behaviour to in-vehicle technology, based on the presence of a voice, and begin to consider the implications that this may have on their interactions with the host device, we selected a ubiquitous example of an in-vehicle speech-based interface - the navigation device ('satnav'). We devised a bespoke questionnaire survey, and invited respondents to self-report their behaviour when using a satnav. We then conducted ethnographicstyle observations documenting the routine use of satnav - from this we hoped to expose illustrative episodes of anthropomorphism, informed by the earlier questionnaire. The paper therefore reports two studies: the method and results are summarised independently for each, whereas the discussion and conclusions are common.

\section{Questionnaire Survey}

The questionnaire survey was designed to explore how people interpret and define their own anthropomorphic behaviour when driving and using a navigation device, and document their attitudes towards this type of behaviour when displayed by others. In addition, it aimed to explore the factors that may influence this behaviour (e.g. the nature of the voice delivering the navigation messages, the style of delivery, the level of trust that drivers placed in the device, usage occasion and duration etc.). Given this broad scope, a bespoke questionnaire was devised, comprising items selected from relevant scales and questionnaires, including the trust in automation scale [19], the anthropomorphic tendencies scale [8], the 'Godspeed' questionnaire [20] and human personality constructs [21]. All questions were presented as seven-point Likert scales, comprising a stem statement, such as "I can trust the satnav", followed by numerically assigned scale points anchored with verbal response descriptors - typically, 'strongly agree' and 'strongly disagree', or 'not at all' and 'completely'. Respondents were asked to indicate their response by selecting a number on the Likert scale that best indicated their level of agreement with each statement. In addition, several open questions inviting written responses were included (e.g. asking respondents to elucidate their selection of voice or name for the device). Ratings underwent multivariate analysis to reveal any significant relationships. Only using selected items from the aforementioned scales may have contravened the analytic techniques specified by each questionnaire, however, the 
intention was to gain a general understanding of the incidence of anthropomorphism, and the attitudes displayed by respondents, to inform subsequent research activities.

\subsection{Method}

The survey was created online, with details posted on a number of online discussion forums and consumer groups. Details were also distributed via email to students and staff at the University of Nottingham. As an incentive to take part, and to encourage respondents to complete the survey (taking approximately 10 and 15-minutes), a donation of $£ 1$ (GBP) was made to a UK registered charity, selected from a shortlist by the respondent, for each completed questionnaire. Only complete datasets were considered in the analysis. Two hundred and eight-five respondents completed the online survey (228 male, 57 female; mean age 44.3 years, range 18-74 years). All participants were experienced UK drivers (mean years with licence: 24.0; mean annual mileage: 16,150), and regular users of satnavs (mean number of years: 3.45). Almost $90 \%$ of respondents used portable devices, comprising dedicated units (79.9\%) and smartphone applications (9.7\%); the remainder used factory-fitted devices. All respondents received directions using voice messages, with some supplementing these with visual iconography.

\section{$2.2 \quad$ Results}

Drivers generally placed high levels of trust in their satnavs. Multivariate analysis revealed that the level of trust was influenced by the length of time that drivers had used the device (Beta $=.20, p=.001)$ and their annual mileage (Beta $=-.13, p=.03$ ), with the highest trust indicated by drivers who had used the devices for the longest. There was evidence to suggest that respondents associated 'human' qualities with their satnav, although some of the findings were inconclusive. For example, it is interesting to note that while respondents generally stated that they would not 'praise' their satnav when it performed well, responses suggest a greater likelihood that they would reprimand the device if it 'made a mistake'.

Almost a quarter of respondents $(\mathrm{n}=66)$ stated that they would give their satnav a name (it was evident from the use of 'he' or 'she' in the written responses that respondents also tended to assign a gender to the device). Many of the names were inspired by human names and characteristics (e.g. 'Bossy Betty', 'Naggy Nora', 'Silicon Sal', 'Sally Satnav' and 'Suzy Satnav'), while some names were inspired by the device manufacturer or the term 'Satnav', with several mildly derogatory derivatives (e.g. 'Sat Nag', 'Nagman'). Drivers who gave their satnav a name also tended to rate statements relating to their own engagement in anthropomorphic behaviour more highly. However, multivariate analysis revealed that the most significant predictor of anthropomorphic tendency was gender, with females rating their own engagement in anthropomorphism more positively than male respondents (Beta $=.29, p<.05$ ). Even so, females also made lower ratings regarding the 'acceptability' of anthropomorphic behaviour displayed by others (Beta $=-.17, p=.008)$. Driving experience was also a predictor of anthropomorphic tendency $(\operatorname{Beta}=-.36, p=.04)$, with responses suggesting that more experi- 
enced drivers were less inclined to engage in anthropomorphic behaviour directed towards their satnav. There was also evidence of anthropomorphism revealed through respondents' comments: "it was only doing this that I realised how often I talk to it"; another stated: "I often shout at my satnav when it goes wrong".

Almost eighty percent of respondents $(n=224)$ chose a female voice to guide them (although this may have been influenced by the system default); only 13 female drivers indicating a preference towards a male voice. Several respondents $(\mathrm{n}=18)$ indicated that they used a voice based on a famous character or celebrity. In these situations, the most popular celebrity voice was John Cleese. Other celebrity voices included Joanna Lumley, Kim Cattrall, Homer Simpson, Mr T and the Queen. Drivers who used a celebrity voice often tended to also refer to the device with that name.

Most respondents identified aspects of human personality associated with their navigation voice, based on both the 'dominance' and 'affiliation' dimensions. Additionally, navigation voices were generally considered to be 'natural' and 'humanlike'. However, higher ratings for these qualities may simply reflect designers' ability to reproduce authentic human voices within the technology. Perhaps most revealing were the written responses when drivers were asked if they would change the navigation voice they used. Most respondents $(\mathrm{n}=268)$ indicated that they would not change the voice. While many citied practical reasons such as, "clarity", or felt that their current navigation voice presented, "minimal distraction", other responses were more revealing. For example: "Joyce is now a family member-you wouldn't change the voice of a family member", "she sounds nice", "[it's a] more human female voice", and "the familiarity with that voice provides reassurance and makes it feel more natural somehow". However, several respondents indicated that they would change the voice depending on context or occasion of use, stating that they downloaded character or celebrity voices to guide them. A variety of reasons were provided for such changes: "after a while the voice gets annoying or boring and I find it better to keep it changing to keep me noticing it", "depends on my mood", "it is fun to change the voices" and "when on journeys with my children I often select a funny voice, dalek, pirate, Darth Vader etc."

\section{Ethnographic Observations}

Ethnography emerged as a method for social inquiry from anthropological roots in the early 1920s, and is popularised today as a method to observe everyday people, undertaking everyday activities, in everyday settings [22]. Within the field of HCI, ethnography has been used to uncover the sort of practical ways that people overcome problems when interacting with a computer, and to reveal how they configure and adapt technology for specific tasks and contexts of use [23]. Findings have been consequently used to inform the design of future technology to ensure that it is applicable to the real world. In its simplest form, undertaking ethnography work involves observing people in a naturalistic setting. 


\subsection{Method}

During the current study, observations took place in the UK, France and Southern Ireland, although all drivers and passengers were from the UK. Participants were contacted personally and asked if they were interested in taking part in the study. Fourteen people were subsequently recruited. These comprised a number of different social groups and use cases, including: two work colleagues travelling to a business meeting, a family of four embarking on a shopping trip in the UK, and journeys associated with two family holidays (with each family unit comprising four members) - holiday travel included travelling to the destination by car (in France and Southern Ireland) and daily excursions while there. All observations took place within the drivers' own vehicles and in most cases involved navigation devices owned by the participants (for one observation, a navigation system was provided, although the participants already had extensive experience using such devices). Only experienced drivers took part (mean years with driving licence: 22.8), with ages ranging from 38 to 45 years (mean age: 41 years). Interactions were documented using an audio recorder operated by each participant during their journeys in which a satnav was used as a matter of course. In total, over thirteen hours of dialogue were recorded. All interactions were transcribed using simplified CA conventions to enable subsequent thematic analysis.

\subsection{Results}

Transcripts were coded using thematic analysis, with the aim of identifying, analysing and reporting patterns (or themes) within the data. A common theme that emerged during the analysis was that drivers and passengers responded to and interacted with the satnav as if it had human qualities. Indeed, people talked to the device, shouted at it, gave it a name, and attempted to evoke it in conversational dialogue. They were also courteous during interactions, offering salutations ("Bye, Jane") and even apologising to the device ("Sorry love, we're having a rest"). People also discussed the satnav as if it were somebody else present, referring to it as 'he' or 'she' ("Where is he taking us? Yeah, alright. Oh dear, he's got a bit chatty now"). In fact, people generally attended to the satnav in a similar manner to how they responded to each other in the car, and applied the same social etiquette, for example, employing conversational turn-taking. Moreover, this behaviour was commonplace and unremarkable - other car occupants did not respond to its peculiarity.

People also appeared to assign more complex human characteristics, behaviours and motivations to the devices, praising it when they successfully reached their destination (Good job, Jane), or reprimanding it if it failed to support them ("She was nagging us through the forest. That was a bad thing, Jane"), as if it intended to act as it did. People even appeared to endow the device with the power of independent thought ("It still thinks we're in Beeston"), human emotions ("She hates it when you come off at the services"), and an awareness of the environment that far exceeded the device's technical capability ("He obviously heard what I said. See, that's what I wanted the first time"). 
There was evidence throughout the observations that it was the voice and the style of delivery that encouraged drivers and passengers to respond to the device in this manner. The voice was not only seen as a medium for delivering navigational instructions - in keeping with human-human interactions, the presence of a voice also appeared to encourage social interaction. The characteristics of the voice were used to assign attributes, such as 'gender' and 'personality' that subsequently defined and determined responses (similar to attributions made during human-human interactions). Some voices therefore encouraged stereo-typical responses from drivers and passengers, for example affecting trust ("Well, I had a female voice on it...It was extremely posh...And just didn't feel like, well, just didn't feel like I could believe her").

The presence of a voice clearly influenced what participants thought about the device. For instance, participants appeared to believe that they were interacting with an intelligent device whose 'motivations' were the same as theirs. This may explain why some people appeared willing to place their trust in what was being said and accept routing errors as genuine mistakes. The voice may have also influenced how drivers used and relied on the support the device provided. Indeed, there were instances when drivers appeared to contemplate changing their normal behaviour, for example by contravening safe driving practices, making illegal manoeuvres, or selecting a road that would normally be deemed to be inappropriate ("Breaking the law... I could just park here and we could walk?...No. No. Go on...Put your foot down. Follow the [satnav]").

\section{Discussion}

Although only limited evidence of anthropomorphism could be gleaned from the questionnaire (possibly due to limitations and biases in this approach [24]), there were abundant examples from the observations of participants interacting with and referring to the satnav as if it had humanlike qualities, and endowing it with human traits, such as a name, gender and personality. To help explain and interpret this behaviour, a number of theses can be offered. The familiarity thesis, for example, offers a cognitive motivation for anthropomorphism, and posits that anthropomorphism allows humans to explain things that they do not understand in terms of things that they do understand (i.e. themselves) [25]. An alternative explanation is offered by the comfort thesis, which proposes that anthropomorphism derives from an emotional motivation, arguing that people anthropomorphise because they are uncomfortable with things that are not like them and 'making' things more like them reduces that discomfort [25]. Moreover, in the face of chronic uncertainty about the nature of the world, guessing that something is humanlike, or has a human cause, constitutes a 'good bet'. If this assumption is found to be true, there is much to be gained, whereas if it is wrong, usually little is lost [25].

While these theories may go some way to explaining the observed behaviours, a more likely explanation is that drivers and passengers were using their experience of being human to define the social structure of the interaction. The Species-Specific Group-Level Coordination System [26], also referred to as the 'Social' Thesis [27], suggests that attributing human characteristics to non-human artefacts changes the value we place on them, and thus defines how we can behave towards them. The social 
thesis therefore claims that the act of anthropomorphising possesses the potential for social consequence [26]. Indeed, evidence from the observations, in particular, suggests that the satnav played an important role in the social fabric in which it was situated. Moreover, drivers (and indeed, passengers) were prepared to afford it high social status, and interacted with it as if it were another human entity (as evidenced by the adoption of social norms and etiquette, such as politeness and turn-taking, when interacting with the device). This might explain why drivers and passengers were quick to praise, or indeed, reprimand the satnav, depending on its performance.

This behaviour is likely to have been inspired by the presence of a 'human' voice, which activated evolutionary instincts [16], inspiring ascriptions of gender and personality, and leading to expectations of 'intelligence' and 'awareness'. However, this naturally raises concerns. The motivation behind using a voice to direct drivers is to ensure that instructions are clear and understandable, and do not distract drivers. The evidence from the study suggests that it also has the potential to inspire unrealistic expectations and inappropriate allocations of trust and reliance. Indeed, during the observations, participants were quick to trust the satnav (sometimes apparently against their better judgement), and there have been many similar anecdotal accounts of poorly calibrated trust in satnavs reported elsewhere (whereby drivers have incorrectly followed instructions, often with dire results ) - it is possible that these have occurred due to inappropriate and unrealistic expectations of 'intelligence' inspired by the perceived 'human' qualities and capabilities.

Nevertheless, a fundamental question posed by the research is whether the participants genuinely and sincerely believed that the satnav had human qualities - the language they used to refer to the device, for example, may simply have been chosen for convenience, in the same way that people routinely use language and terminology to refer to their daily interactions with computers and technology that are taken directly from humans (e.g. becoming 'infected with a virus', being user-'friendly' or 'thinking' while a particularly complex program is being executed [28]). However, anthropomorphism makes no claims that it is the same as relating to other people: "we do not impute human personality in all its subtle complexity; we paint with broad strokes, thinking only of those traits that are useful to us in the particular context" [29]. Therefore, rather than dismissing anthropomorphism as a probable explanation for the observed behaviours and responses, it is suggested that the findings instead reinforce this view, drawing particular attention to the peculiarities and character of modern day anthropomorphism, as well as revealing some of the potential pitfalls in this context.

Finally, it is worth noting that, although responses were actually collected during 2010 , the structure and delivery of navigational voice messages, and the front-end of satnavs, remain largely unchanged today, despite technological advancements in the underpinning technology. Moreover, with the current resurgence in speech interfaces, and the expectation that speech is likely to play an important role in supporting drivers through increasing levels of automated driving in the near-future [30,31], findings are likely to be highly relevant today. The results can be used to inform the design and development of future in-vehicle speech-based technology, and suggest that, on the one hand, designers must be mindful of drivers' automatic attributions associated with 'natural' methods of interaction, such as speech, and the potential impact that this may have 
on their behaviour (particularly in terms of inappropriate allocation of trust and overreliance on the technology). On the other hand, knowledge on this topic can be used to enhance the user experience and acceptance/uptake of speech-based technology in the automotive domain (and indeed, elsewhere).

\section{Conclusion}

A questionnaire survey enriched by ethnographic-style observations explored the propensity of drivers (and passengers) to engage in anthropomorphic behaviour during the routine use of an in-vehicle navigation device. Although, on face value, some of the self-reported behaviour of the questionnaire respondents was unremarkable, further interrogation of the data revealed subtle behaviours traditionally considered exclusive to humans, such as naming the device, talking to it and blaming it when mistakes were made. The observations were far more revealing, with evidence of anthropomorphism common and widespread, including making social reference to the device (using 'he' or 'she'), attempting to engage it in discussions, reprimanding it (following erroneous instructions or navigational errors) and praising it after successfully reaching a destination. Moreover, this behaviour was evident during routine interactions, was not dependent on occupancy or occasion use, and was not highlighted by other occupants as unusual or abnormal. The results can be used to inform the design and development of future in-vehicle speech-based technology.

\section{References}

1. Ghazizadeh, M., Lee, J.D. and Boyle, L.N., 2012. Extending the Technology Acceptance Model to assess automation. Cognition, Technology \& Work, 14(1), pp.39-49.

2. Schade, J. and Baum, M., 2007. Reactance or acceptance? Reactions towards the introduction of road pricing. Transportation Research Part A: Policy and Practice, 41(1), pp.41-48.

3. The Free Dictionary, "The Free Dictionary," Online.. Available: http://www.thefreedictionary.com/anthropomorphism. Accessed 03042017.

4. DiSalvo, C. and Gemperle, F., 2003, June. From seduction to fulfillment: the use of anthropomorphic form in design. In Proceedings of the 2003 international conference on Designing pleasurable products and interfaces (pp. 67-72). ACM.

5. Caporael, L.R., 1986. Anthropomorphism and mechanomorphism: Two faces of the human machine. Computers in Human Behavior, 2(3), pp.215-234.

6. Daston, L. and Mitman, G. eds., 2005. Thinking with animals: New perspectives on anthropomorphism. Columbia University Press.

7. Robert, F. and Robert, J., 2000. Faces. Chronicle Books. NY

8. Chin, M.G., Sims, V.K., Clark, B. and Lopez, G.R., 2004, September. Measuring individual differences in anthropomorphism toward machines and animals. In Proceedings of the human factors and ergonomics society annual meeting (Vol. 48, No. 11, pp. 1252-1255). Sage CA: Los Angeles, CA: SAGE Publications.

9. DiSalvo, C., Gemperle, F. and Forlizzi, J., 2005. Imitating the human form: Four kinds of anthropomorphic form. Unpublished manuscript. Accessed April.

10. Mitchell, R.W., Thompson, N.S. and Miles, H.L. eds., 1997. Anthropomorphism, anecdotes, and animals. SUNY Press. 
11. Choi, J. and Kim, M. 2009. Anthropomorphic Design: Projecting Human Characteristics to Products, in International Association of Societies of Design Research Conference (IASDR2009), Seoul, Korea.

12. Shneiderman B. and Plaisant C., 2004. Designing the User Interface: Strategies for Effective Human-Computer Interaction, 4th ed., London: Pearson Addison Wesley.

13. Barthes, R., 1977. Music Image Text. New York: Hill and Wang.

14. Chion, M., 1999. The voice in cinema. Columbia University Press.

15. Nass, C. and Brave, S., 2005. Wired for speech: How voice activates and advances the human-computer relationship. MIT press.

16. Large, D.R., Burnett, G.E., 2013. Drivers' preferences and emotional responses to satellite navigation voices. International Journal of Vehicle Noise and Vibration, 9(1-2), pp.28-46.

17. Barón, A. and Green, P., 2006. Safety and usability of speech interfaces for in-vehicle tasks while driving: A brief literature review (No. UMTRI-2006-5). University of Michigan, Transportation Research Institute.

18. Large, D.R., Burnett, G., Antrobus, V., Skrypchuk, L. 2018. Driven to Discussion: Engaging Drivers in Conversation with a Digital Assistant as a Countermeasure to Passive Task-Related Fatigue. IET Intelligent Transport Systems (in press)

19. Jian, J.Y., Bisantz, A.M. and Drury, C.G., 1998, October. Towards an empirically determined scale of trust in computerized systems: distinguishing concepts and types of trust. In Proceedings of the Human Factors and Ergonomics Society Annual Meeting (Vol. 42, No. 5, pp. 501-505). Sage CA: Los Angeles, CA: SAGE Publications.

20. Bartneck, C., Croft, E. and Kulic, D., 2008, March. Measuring the anthropomorphism, animacy, likeability, perceived intelligence and perceived safety of robots. In Metrics for HRI workshop, technical report (Vol. 471, pp. 37-44).

21. Kiesler, D.J., 1983. The 1982 interpersonal circle: A taxonomy for complementarity in human transactions. Psychological review, 90(3), p.185.

22. Crabtree, A., 2006. Designing collaborative systems: A practical guide to ethnography. Springer Science \& Business Media.

23. Schensul, S.L., Schensul, J.J. and LeCompte, M.D., 1999. Essential ethnographic methods: Observations, interviews, and questionnaires (Vol. 2). Rowman Altamira.

24. Couch, A. and Keniston, K., 1960. Yeasayers and naysayers: Agreeing response set as a personality variable. The Journal of Abnormal and Social Psychology, 60(2), p.151.

25. Guthrie, S.E. and Guthrie, S., 1993. Faces in the clouds: A new theory of religion. Oxford University Press on Demand.

26. Caporael, L.R. and Heyes, C.M., 1997. Why anthropomorphize? Folk psychology and other stories. Anthropomorphism, anecdotes, and animals, pp.59-73.

27. DiSalvo, C. and Gemperle, F., 2003, June. From seduction to fulfillment: the use of anthropomorphic form in design. In Proceedings of the 2003 international conference on Designing pleasurable products and interfaces (pp. 67-72). ACM.

28. Branscomb, L.M. 1979. The human side of the computer. Paper presented at the Symposium on Computer, Man and Society, Haifa, Israel.

29. Laurel, B., 1997. Interface agents: Metaphors with character. Human Values and the design of Computer Technology, pp.207-219.

30. Large, D.R., Clark, L., Quandt, A., Burnett, G. and Skrypchuk, L., 2017. Steering the conversation: a linguistic exploration of natural language interactions with a digital assistant during simulated driving. Applied ergonomics, 63, pp.53-61.

31. Eriksson, A. and Stanton, N.A., 2017. The chatty co-driver: A linguistics approach applying lessons learnt from aviation incidents. Safety science, 99, pp.94-101. 